\title{
FABRICATION AND CHARACTERIZATION OF RALOXIFENE LOADED SOLID-LIPID NANOPARTICLES
}

\author{
NAVIN CHANDRA PANT ${ }^{*}$, VIJAY JUYAL ${ }^{1}$
}

1Department of Pharmaceutical Sciences, Bhimtal Campus, Kumaun University, Nainital

*Email: navpant@gmail.com

Received: 11 Apr 2021, Revised and Accepted: 24 May 2021

\begin{abstract}
Objective: The poor water solubility of the drug presents a great challenge for the formulation development and results in low oral bioavailability. The oral bioavailability of Raloxifene HCl (RLX) is very low $(<2 \%)$ in humans due to its poor solubility. The objective of the present study was to develop RLX loaded solid-liquid nanoparticles for effective drug delivery.
\end{abstract}

Methods: Compritol 888 ATO-based RLX-loaded solid lipid nanoparticles (SLNs) were formulated using the oil in water microemulsion method. Drug-excipients compatibility was confirmed through Fourier transform infrared spectroscopy, Differential scanning calorimetry methods. The SLN was characterized for particle size, surface morphology, entrapment efficiency.

Results: A total of seventeen formulations (SLN1-SLN17) were developed as per the 3 levels 3 factor Box-Behnken design. The model used for the analysis was statistically analyzed using ANOVA and the goodness of fit was evaluated using diagnostic plots. As per the response-surface plots, the amount of lipid, poloxamer 407, and ultrasonication time have a significant effect on the particle size and entrapment efficiency (\%EE). The developed RLX-loaded SLNs have the size and \%EE in the range of $165.63 \pm 2.62 \mathrm{~nm}$ to $315.33 \pm 4.87 \mathrm{~nm}$ and $75.21 \pm 2.32 \%$ to $95.32 \pm 2.11 \%$. The TEM analysis showed that the developed RLX-loaded SLNs were almost spherical and has a small size range.

Conclusion: The high biocompatibility, biodegradability, ability to protect drugs in GIT, and sustained release properties make SLNs an ideal candidate to resolve poor oral bioavailability challenges.

Keywords: Nanoparticles, Drug delivery, Raloxifene, Solid-lipid nanoparticles, Box-Behnken design

(C) 2021 The Authors. Published by Innovare Academic Sciences Pvt Ltd. This is an open access article under the CC BY license (https://creativecommons.org/licenses/by/4.0/) DOI: https://dx.doi.org/10.22159/ijap.2021v13i4.41774. Journal homepage: https://innovareacademics.in/journals/index.php/ijap

\section{INTRODUCTION}

Raloxifene hydrochloride (RLX) is a second-generation selective estrogen receptor modulator (SERM) that has anti-estrogenic effects on breast and uterine tissues, and estrogenic effects on bone, lipid metabolism, and blood coagulation [1] It is considered first-line therapy for the management of postmenopausal osteoporosis [2]. Poor solubility of the RLX $(0.25 \mathrm{mg} / \mathrm{lit})$ results in only $60 \%$ absorption of the administered dose and poor oral bioavailability (less than 2\%). In addition, extensive first-pass metabolism of RLX by glucuronide conjugation also results in poor oral bioavailability [3]. The RLX comes under class II (low solubility and high permeability) of biopharmaceutical classification [4]. The poor water solubility and poor oral bioavailability present a continuous challenge for the formulation development and effective treatment of the diseases. Therefore there is an unmet need for an effective delivery system that can overcome the existing challenges.

To date, solid-lipid nanoparticles (SLNs) have emerged as a potential carrier that can enhance the solubility and oral bioavailability of poorly water-soluble drugs $[5,6]$. SLNs have a lipid core that mimics the chylomicron formation and along with the loaded drug it absorbs through the transcellular mechanism of lipid absorption [7]. In addition to their small size, the SLNs have combined property of a lipid emulsion and the polymeric nanoparticle with enhanced biocompatibility, biodegradability, ability to protect drugs in the gastrointestinal tract (GIT) from the harsh environment, sustained drug release, and rapid uptake $[8,9]$. Studies have shown that the RLX loaded into the triglyceride-based SLNs has improved oral bioavailability compared to a free RLX [10]. However, studies have shown that the SLNs prepared with triglycerides with shorter carbon chain lengths have some limitations for oral administration due to their susceptibility towards intestinal lipase and co-lipase enzyme system [11]. However, SLNs prepared with lipid having a higher carbon chain, such as Compritol 888 ATO (glyceryl behenate) are more resistant towards the enzyme system. Moreover, SLNs prepared with stabilizers, such as poloxamers have longer circulation duration in the body reduced protein adsorption, and low phagocytic uptake [12].

In the present investigation, we used the Box-Behnken design (BBD) for the optimization of RLX-loaded SLNs. The effect of variables on the properties of the SLNs was evaluated using contour and response-surface plots. We selected lipid, poloxamer 407 (P407), and sonication time as a variable and particle size and entrapment efficiency (EE) as a response. The BBD design was selected as it requires few runs and is particularly useful when extreme treatment combinations need to be avoided. We have investigated the drugexcipients compatibility by Fourier transform infrared (FTIR) spectroscopy, Differential scanning calorimetry (DSC) The model used for the optimization was analyzed statistically via ANOVA, and the goodness of fit was evaluated through different diagnostic plots. The optimized condition was selected based on the desirability function value and the fresh formulation was developed to validate the optimized set parameters.

\section{MATERIALS AND METHODS}

\section{Materials}

Raloxifene hydrochloride was obtained as a gift sample from Archerchem Healthcare Pvt India. Poloxamer 407 was purchased from SD fine chemicals, Mumbai, India India. Compritol 888 ATO was obtained as a gift sample from Archerchem Healthcare Pvt India. Dimethyl sulfoxide (DMSO) was purchased from SD fine chemicals, Mumbai, India. All other chemicals are of analytical grade.

\section{Drug-excipients compatibility studies}

Fourier transform infrared spectroscopy

A drug-excipients compatibility study was carried out by Fourier transform infrared spectroscopy (FTIR-8400S, Shimadzu). The FTIR spectrum of the drugs alone and physical mixtures (RLX+Comptrol and $\mathrm{RLX}+\mathrm{P} 407)$ were recorded using the potassium bromide (KBr) dispersion method. The test samples were added to $\operatorname{KBr}(1: 1)$ and 
examined at the range of 400 to $4000 \mathrm{crr1}$ with a resolution of 4 $\mathrm{cm}-1$.

\section{Differential scanning calorimetry}

For differential scanning calorimetry (DSC), thermograms of the samples (RLX, RLX+RLX+Comptrol, and RLX+P407) were obtained by using a differential scanning calorimeter (TA Instruments $Q$ 2000). Samples were weighed directly in pierced DSC aluminum pan and scanned in the temperature range of $25-300^{\circ} \mathrm{C}$ under an atmosphere of dry nitrogen. A heating rate of $10{ }^{\circ} \mathrm{C} / \mathrm{min}$ was used and thermograms obtained were observed for interaction between drug and excipients.

\section{Design of experiment}

For the optimization of RLX-loaded SLNs, 3-factor, 3-level BoxBehnken design (total of 17 experimental runs with 5 center points) was used to analyze the overall effect of the variables on the properties of SLNs. The lipid amount (X1), surfactant concentration (X2), and sonication time (X3) were selected as variables. The particle size (Y1) and entrapment efficiency (Y2) were selected as a response. The responses were analyzed using Design-Expert software (Trial version 11.0.5.0, Stat-Ease Inc., MN). The levels of variables and responses used for the optimization of RLX-loaded SLNs were illustrated in table 1.

Table 1: Design of experiment for the optimization of RLX-loaded SLNs

\begin{tabular}{|c|c|c|c|}
\hline \multicolumn{4}{|l|}{ Independent variables } \\
\hline & Low $(-1)$ & Medium (0) & High (+1) \\
\hline Compritol 888 ATO (mg) & 500 & 1000 & 1500 \\
\hline Poloxamer 407 (\% w/v) & 3 & 5 & 7 \\
\hline Ultra sonication time ( $\mathrm{min}$ ) & 5 & 7.5 & 10 \\
\hline \multicolumn{4}{|l|}{ Dependent variables } \\
\hline Particle size $(\mu \mathrm{m})$ & Minimum & & \\
\hline Entrapment efficiency (\%) & Maximum & & \\
\hline
\end{tabular}

\section{Preparation of RLX-loaded SLNs}

The RLX-loaded SLNs were prepared via an oil-in-water microemulsion technique with some modification [13]. Briefly, Compritol 888 ATO was heated to above its melting point and the RLX (50 mg) was dispersed into the melted lipid. In parallel, an aqueous solution of P 407 was prepared and heated to the same temperature as the lipid-drug dispersion. When both the solution become isothermal, the aqueous solution was added to the lipid phase followed by homogenization (Polytron PT 3100D, Kinematica, Lucerne, Switzerland) at 10,000 rpm for $10 \mathrm{~min}$. The developed microemulsion was quickly sonicated probe sonicator (Vibra cell, Sonics, USA) for a predetermined period. In the end, the developed formulation was stirred $(50 \mathrm{rpm})$ in ice-cold water for about 10 min for the hardening of nanoparticles. The developed SLNs were then used for further analysis.

\section{Formulation characterization}

\section{Particle size and zeta potential}

Particle size, polydispersity index (PDI), and zeta potential (ZP) of the RLX-loaded SLN were measured by Zetasizer Nano ZS 90 instrument (Malvern Instruments, Worcestershire, UK). The intensity of scattered light was measured at an angle of $90^{\circ}$. The samples were appropriately diluted with double-distilled high purity water before measurement.

\section{Surface morphology}

The morphology of the developed RLX-loaded SLNs was analyzed using Transmission electron microscopy (TEM, FEI Tecnai G220 STwin TEM). The prepared RLX-loaded SLNs were dispersed in distilled water and a drop of dispersion was placed on a carboncoated copper grid followed by drying. This grid was mounted in the instrument and photographs were taken at various magnifications.

\section{Entrapment efficiency}

The entrapment efficiency (\%EE) of RXL-loaded SLNs was determined by measuring the concentration of unentrapped drugs in the supernatant of the aqueous medium by centrifugation method. Immediately after the end of the formulation development step, the developed nanosuspension was centrifuged at a speed of $10,000 \mathrm{rpm}\left(\mathrm{C} 4\right.$, Remi) for $15 \mathrm{~min}$ at $4{ }^{\circ} \mathrm{C}$. The supernatant was separated and the amount of RLX in the supernatant was determined using a UV-Vis spectrophotometer (UV1800, Szimadzu) at $286 \mathrm{~nm}$ after appropriate dilution. The percentage entrapment efficiency ( $\% \mathrm{EE}$ ) was calculated by using the following formula,

\section{$\frac{\text { Total drug content-Free drug }}{\text { Total drug content }} \times 100$}

\section{Statistical analysis}

The 3 levels, 3 factors, BBD were used to investigate the effect of variables on the responses of RLX-loaded SLNs [14, 15]. The statistical model used for the design of the experiment for the development of RLX-loaded SLNs was analyzed in terms of fit summary, lack of fit test, the sum of the square, correlation coefficient $\left(\mathrm{R}^{2}\right)$, adjusted $\mathrm{R}^{2}$, and predicted $\mathrm{R}^{2}$. The selected model used to evaluate the effect of variables on responses was analyzed by ANOVA, $F$-value, $P$-value, lack of fit, Degree of freedom (df), Adjusted $R^{2}\left({ }_{\text {Adj }} R^{2}\right)$ and Predicted $R^{2}\left({ }\right.$ red $\left.R^{2}\right)$. The goodness of fit of the proposed model was investigated by plotting diagnostic plots, such as externally studentized residuals vs. predicted plot, predicted vs. actual plot, normal probability plot, and externally studentized residuals vs. run number plot. The effect of variables on the responses was evaluated with the help of response surface plots (3D) and contour plots (2D). The optimized values of the variables were estimated within the set criterion of desirability and further analyzed through overlay plots. Finally, optimized values of variables were selected for the development of optimized formulation.

\section{RESULTS AND DISCUSSION}

\section{Drug excipients compatibility}

The drug-excipients compatibility study was evaluated using FTIR spectroscopy and DSC. The FTIR spectrum of RLX showed characteristic peaks at $3465.27 \mathrm{~cm}^{-1}$ (O-H stretching), $2938.42 \mathrm{~cm}^{-1}$ (Aromatic C-H bond), $1603.88 \mathrm{~cm}^{-1}$ ( $\mathrm{C}=0$ stretching), $1500.68 \mathrm{~cm}^{-1}$ (Aromatic $\mathrm{C}=\mathrm{C}$ ), $1463.07 \mathrm{~cm}^{-1}$ (-S-benzothiophene), $1263.43 \mathrm{~cm}^{-1}$ (phenolic C-0 stretch) and $1039.68 \mathrm{~cm}^{-1}$ (ether C-O-C stretch) (fig. 1(A)). These characteristic peaks are also visible in the physical mixture of RLX and Compritol 888 ATO, and RLX and P407 (fig. 1 (B and C)

According to the DSC studies, the thermogram of RLX showed a characteristic single sharp peak at $270.66{ }^{\circ} \mathrm{C}$ which indicates the melting point of the drug and its crystalline nature (fig. 2(A)). The thermograms of physical mixtures also show the characteristic peak of RLX without any significant change (fig. 2(B and C)). The results from these studies indicate the drug-excipients compatibility and their suitability of formulation development.

\section{Formulation characterization}

The RLX-loaded SLNs were developed using the microemulsion method. According to the BBD design, a total of 17 formulations (SLN1-SLN17) were developed and characterized for size, ZP, PDI, morphology and \%EE. The developed RLX-loaded SLNs have the size and \%EE in the range of $165.63 \pm 2.62 \mathrm{~nm}$ to $315.33 \pm 4.87 \mathrm{~nm}$ and $75.21 \pm 2.32 \%$ to $95.32 \pm 2.11 \%$ (table 2 ). The zeta potential and PDI of all the formulations were under acceptable limits. The TEM analysis showed that the developed RLX-loaded SLNs were almost spherical in shape and has a small size range (fig. 3). 


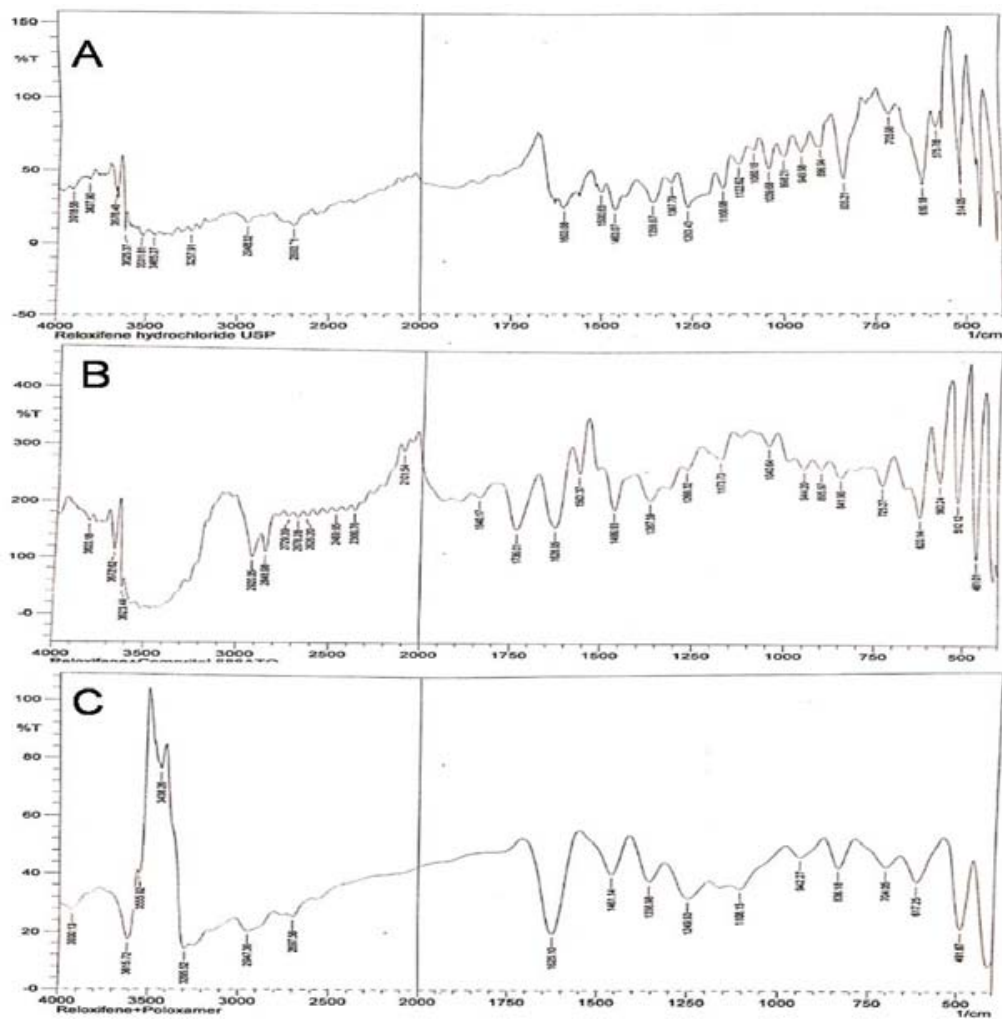

Fig. 1: FTIR spectrum of (A) RLX, (B) RLX+Compritol 888 ATO and (C) RLX+P407
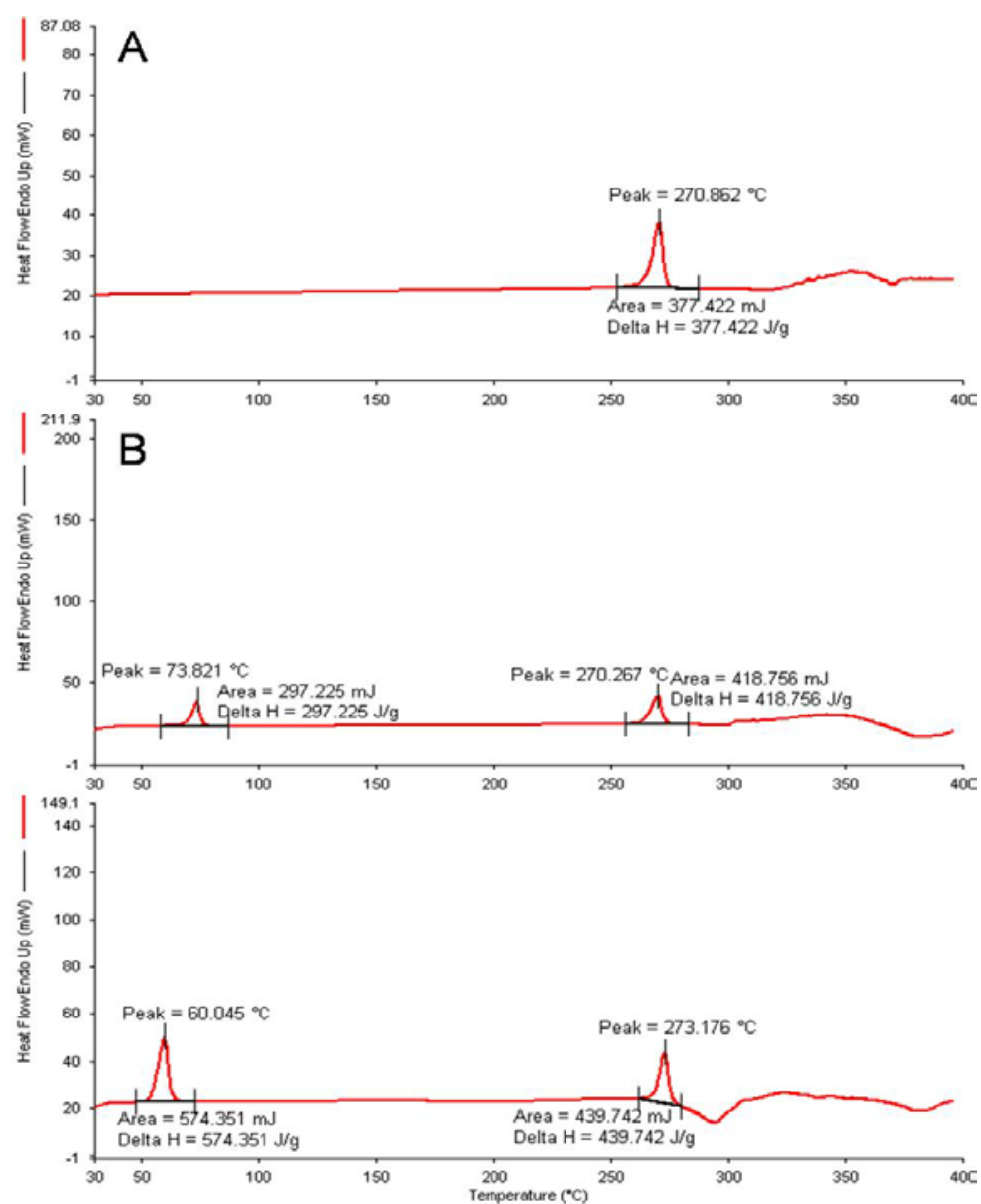

Fig. 2: Thermograph of (A) RLX, (B) RLX+RLX+Compritol 888 ATO and (C) RLX+P407 
Table 2: RLX-loaded SLNs and obtained reponces (mean \pm SD)

\begin{tabular}{|c|c|c|c|c|c|}
\hline \multirow{3}{*}{$\begin{array}{l}\text { Formulation } \\
\text { code }\end{array}$} & Factor 1 & Factor 2 & Factor 3 & Response 1 & Response 2 \\
\hline & A: Lipid & B: P407 & C: Sonication & Size & EE \\
\hline & (mg) & $(\% \mathrm{ov} / \mathrm{v})$ & (min) & (nm) & $(\%)$ \\
\hline SLN1 & 500 & 7 & 7.5 & $228.12 \pm 4.32$ & $76.52 \pm 1.56$ \\
\hline SLN2 & 1500 & 5 & 5 & $238.33 \pm 5.15$ & $95.32 \pm 2.11$ \\
\hline SLN3 & 1000 & 5 & 7.5 & $165.63 \pm 2.62$ & $93.51 \pm 1.98$ \\
\hline SLN4 & 500 & 5 & 5 & $185.32 \pm 2.56$ & $69.21 \pm 1.56$ \\
\hline SLN5 & 1000 & 7 & 10 & $257.65 \pm 3.55$ & $76.87 \pm 2.32$ \\
\hline SLN6 & 1000 & 7 & 5 & $249.21 \pm 4.21$ & $88.43 \pm 2.65$ \\
\hline SLN7 & 1000 & 3 & 10 & $280.43 \pm 5.76$ & $74.21 \pm 1.76$ \\
\hline SLN8 & 1000 & 5 & 7.5 & $168.21 \pm 3.11$ & $91.43 \pm 3.11$ \\
\hline SLN9 & 1000 & 3 & 5 & $284.21 \pm 4.34$ & $75.21 \pm 2.32$ \\
\hline SLN10 & 500 & 3 & 7.5 & $255.55 \pm 3.87$ & $58.54 \pm 1.12$ \\
\hline SLN11 & 1000 & 5 & 7.5 & $169.43 \pm 2.22$ & $90.56 \pm 2.43$ \\
\hline SLN12 & 1500 & 5 & 10 & $245.32 \pm 3.65$ & $86.43 \pm 1.66$ \\
\hline SLN13 & 500 & 5 & 10 & $285.32 \pm 4.78$ & $75.98 \pm 2.31$ \\
\hline SLN14 & 1500 & 3 & 7.5 & $315.33 \pm 4.87$ & $91.21 \pm 3.21$ \\
\hline SLN15 & 1000 & 5 & 7.5 & $167.87 \pm 3.21$ & $91.34 \pm 2.13$ \\
\hline SLN16 & 1000 & 5 & 7.5 & $168.43 \pm 2.87$ & $91.11 \pm 2.34$ \\
\hline SLN17 & 1500 & 7 & 7.5 & $280.23 \pm 4.13$ & $78.32 \pm 1.67$ \\
\hline
\end{tabular}

*Standard deviation, Number of experiments done (n):3

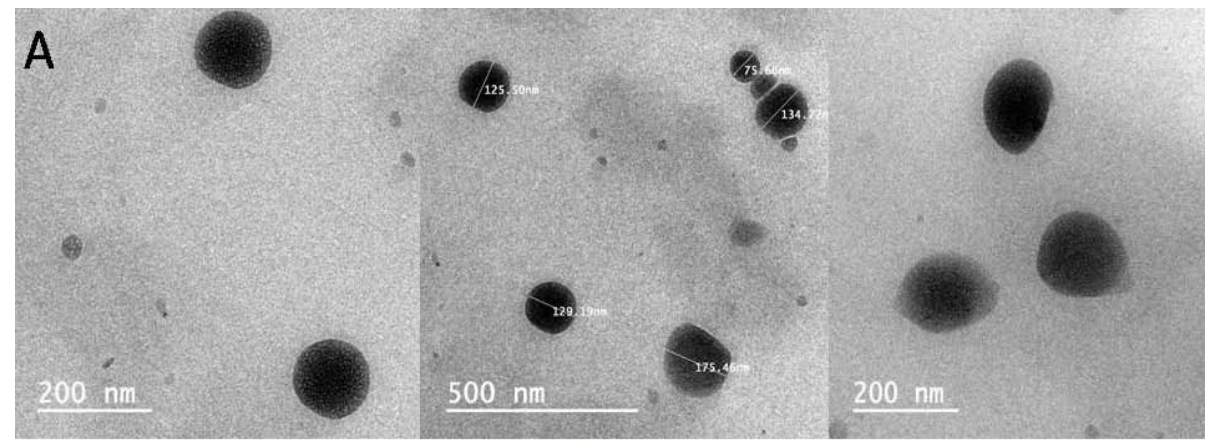

B

Size Distribution by Intensity

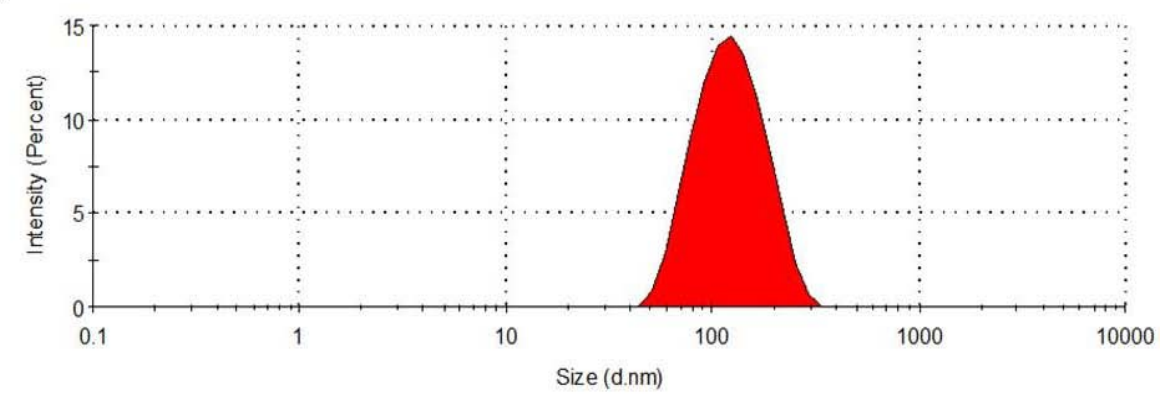

Fig. 3: Characterization of SLNs. (A) TEM images and (B) size distribution graph by zeta sizer

\section{Statistical analysis}

\section{Effect on the size}

According to the fit summary, a sequential model sum of the square, model summary statistics and fit summary details (Predicted $\mathrm{R}^{2}\left(\mathrm{Pred}^{2}\right)$, Adjusted $\mathrm{R}^{2}\left({ }_{\text {Adj }} \mathrm{R}^{2}\right), F$-value, $p$-value, and degree of freedom (df)), a quadratic model was selected to evaluate the effect of variables on the SLNs size. The variables affecting the particle size, such as (A) lipid (B) P407 (C) Sonication time were given by the quadratic equation,

$$
\begin{gathered}
\text { Size }(Y 1)=+167.91+15.61 \mathrm{~A}-15.04 \mathrm{~B}+13.96 \mathrm{C}-1.92 \mathrm{AB}- \\
23.25 \mathrm{AC}+3.05 \mathrm{BC}+36.30 \mathrm{~A}^{2}+65.60 \mathrm{~B}^{2}+34.36 \mathrm{~A}^{2}
\end{gathered}
$$

The model used to evaluate the effect on particle size was analyzed through analysis of variance (ANOVA). The ANOVA showed that the used model was significant with the acceptable model $F$-value (11.97), the $p$-value of $0.0018(P<0.0500)$, degree of freedom (df) of 9 , and lack of fit $F$-values of 429.21 ( $p$-value-0.0001 ( $p<0.0500)$ ). The goodness of fit of the proposed model for the particle size was investigated using diagnostic plots (fig. $4(\mathrm{~A}-\mathrm{C})$ ). The normal plots of residuals showed a majority of the colored points indicating the value of particle size were located around the normal probability straight line. This indicates the normality of residuals and relevant analysis of response data (fig. 4 (A)). The residual vs predicted and residual vs run value plot showed that all the values indicating the particle size lay within the set upper and lower limit. The plot showed a random distribution of the studentized residuals, which indicated that the assumption of constant variance was true (fig. 4 (B and C)). 

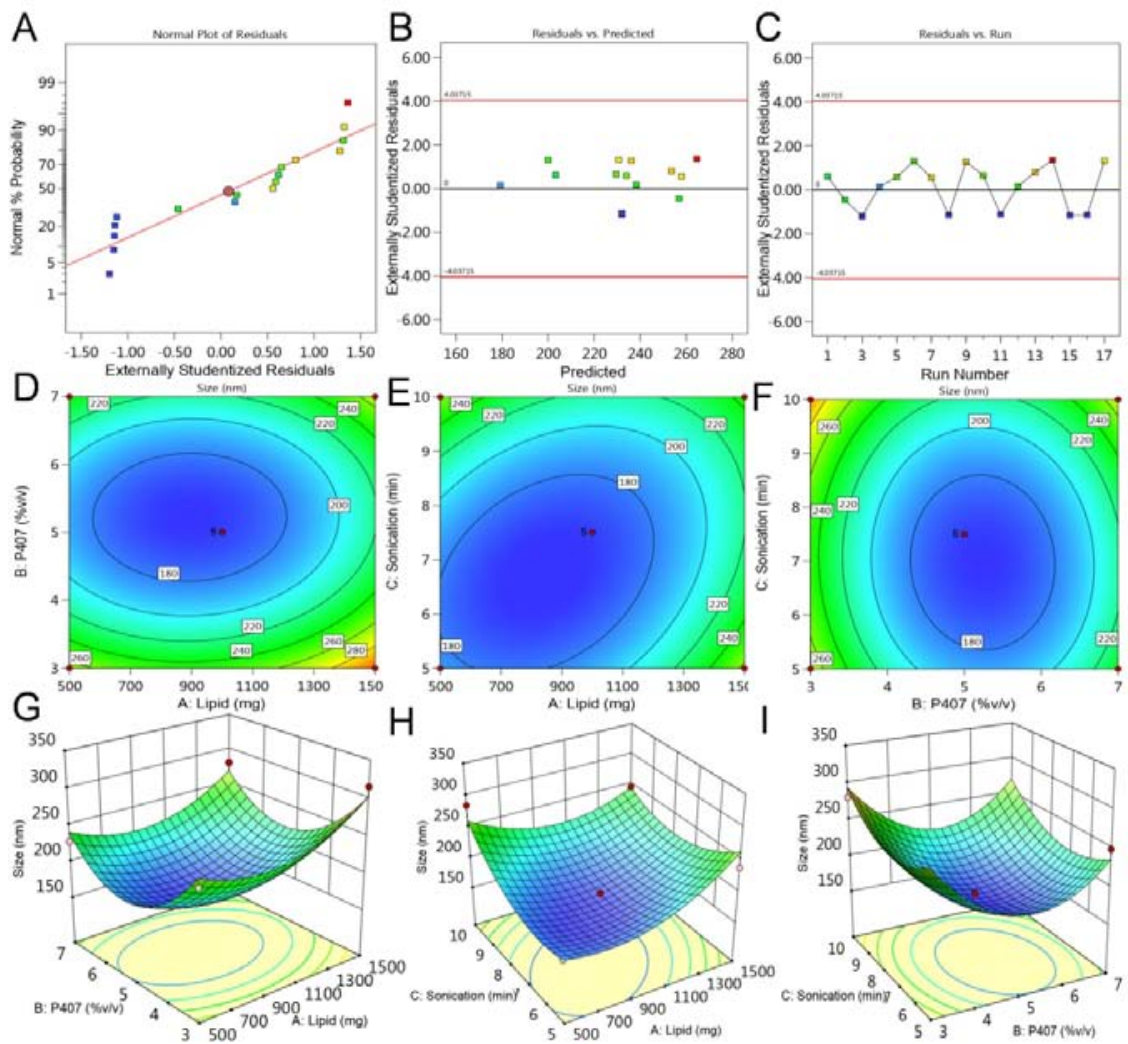

Fig. 4: Effect of variables on size. (A) The normal plot of residuals, (B) residual vs predicted plot, (C) residual vs run, (D-F) 2D contour plots, and (G-I) response surface 3D plots

The effect of variables (A: Lipid, B: P407, and C: Sonication time) on the particle size was evaluated using contour (2D) and Responsesurface (3D) plots. Contour and response-surface plots between lipid and P407, keeping sonication time constant showed an increase in particle size at the high amount of lipid. The reason for the increase in size with the increase in lipid could be due to an increase in the velocity of the dispersion increase with the lipid fraction, result in higher surface tension and ultimately larger particle size. Whereas, with an increase in $\mathrm{P} 407$ concentration there was a drop in particle size, however, with further increase in P407 results in increased particle size (fig. 4(D, G)). The reason for the decrease in the size with increased P407 could be due to a reduction in the interfacial tension more effectively result in smaller particle size [16].

Contour and response-surface plots between the sonication time and lipid keeping P407 constant showed that at the highest value of sonication time and lipid amount the particle size increases (fig. 4(E, $\mathrm{H})$ ). An increase in the particle size at long sonication time could be due to the possible aggregation of small particles into a bigger one that stuck to the wall of the beaker and dried. A sonication-mediated turbulent flow and shocking waves generated by cavitation in liquids irradiated with ultrasound, particles are associated together at extremely high speeds contribute to the facile agglomeration process [17]. A similar effect of sonication time and P407 was also observed in other Contour and response-surface plots (fig. 4(F, I)).

\section{Effect on \%EE}

According to the fit summary, the sequential model sum of a square, model summary statistics and fit summary details (Predicted $\mathrm{R}^{2}$ $\left({ }_{\text {Pred }} \mathrm{R}^{2}\right)$, Adjusted $\mathrm{R}^{2}\left({ }_{\mathrm{Adj}} \mathrm{R}^{2}\right), F$-value, $p$-value, and degree of freedom (df)), a quadratic model was selected to evaluate the effect of variables on the SLNs \%EE. The variables affecting the particle size, such as (A) lipid (B) P407 (C) Sonication time were given by the quadratic equation,

$\% \mathrm{EE}(\mathrm{Y} 2)=+91.59+8.88 \mathrm{~A}+2.62 \mathrm{~B}-1.83 \mathrm{C}-7.72 \mathrm{AB}-3.92 \mathrm{AC}-2.64 \mathrm{BC}-$ $6.19 \mathrm{~A}^{2}-9.25 \mathrm{~B}^{2}-3.66 \mathrm{~A}^{2}$
The model used to evaluate the effect on \%EE was analyzed through analysis of variance (ANOVA). The ANOVA showed that the used model was significant with an acceptable model $F$-value (38.56), the $p$-value of $0.0400(P<0.0500)$, a degree of freedom (df) of 9 , and lack of fit $F$-values of 72.56 ( $p$-value-0.0400 $(p<0.0500)$ ). The goodness of fit of the proposed model for the particle size was investigated using diagnostic plots (fig. 5(A-C)). The normal plots of residuals showed a majority of the colored points indicating the value of particle size were located around the normal probability straight line. This indicates the normality of residuals and relevant analysis of response data (fig. 5(A)). The residual vs predicted and residual vs run value plot showed that all the values indicating the particle \%EE lay within the set upper and lower limit. However, only two points were found outside the limit range. The plot showed a random distribution of the studentized residuals, which indicated that the assumption of constant variance was true (fig. $5(B$ and $\mathrm{C})$ ).

The effect of variables (A: Lipid, B: P407, and C: Sonication time) on the particle \%EE was evaluated using contour (2D) and Responsesurface (3D) plots. Contour and response-surface plots between lipid and P407, keeping sonication time constant showed an increase in \%EE with an increase in the amount of the lipid. The increase in \%EE with lipid is could be due to the increased viscosity of the medium because increasing the amount of lipid resulted in faster solidification of the nanoparticles. This would also prevent drug diffusion to the external phase of the medium $[18,19]$. However, there was a slight increase in \%EE with the increase in P407 amount (fig. 5(D, G)).

Contour and response-surface plots between the sonication time and lipid keeping P407 constant showed a significant increase in $\% \mathrm{EE}$ with the increase in lipid amount. The sonication time has no significant effect on the \%EE (Fig.5 (E, H)). Contour and responsesurface plots between the sonication time and P407 showed an increase in \%EE with the increase in P407 concentration. The sonication time has no significant effect on the \%EE (fig. 6(F, I)). 

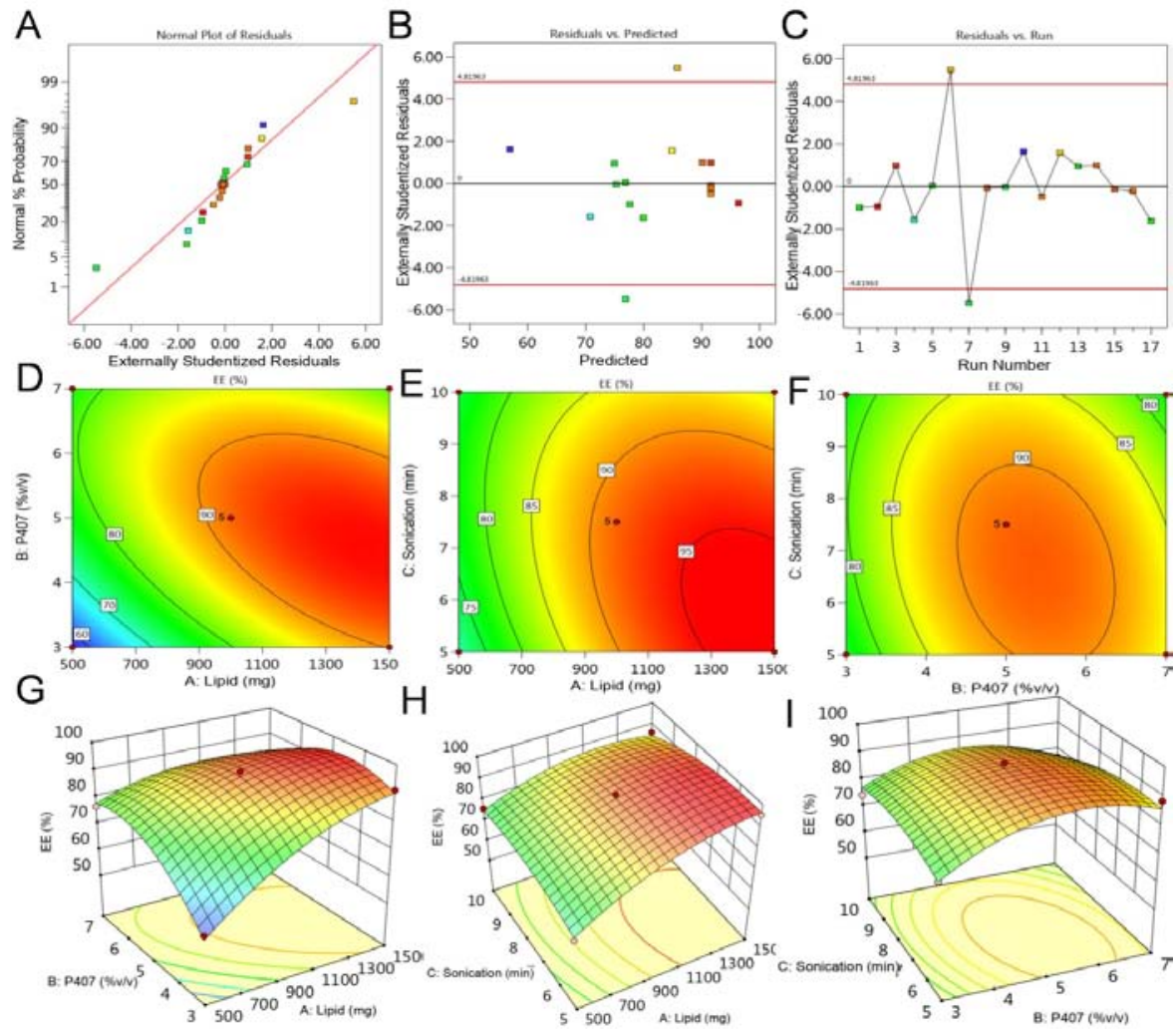

Fig. 5: Effect of variables on entrapment efficiency. (A) The normal plot of residuals, (B) residual vs predicted plot, (C) residual vs run, (DF) 2D contour plots, and (G-I) response surface 3D plots

\section{CONCLUSION}

The Poor water solubility of the drug not only affects its oral bioavailability and overall therapeutic effects, however, but also presents a great challenge for formulation development. In form past decade, SLNs have emerged as a potential system that can effectively overcome these limitations. The high biocompatibility, biodegradability, ability to protect drugs in GIT, and sustained release properties make SLNs an ideal candidate to resolve poor oral bioavailability challenges. In the present investigation, Compritol 888 ATO-based SLNs were successfully prepared via emulsion technique. The effect of variables on the properties of the RLXloaded SLNs was analyzed using BBD. The formulations were optimized via the desirability function which gives a set of conditions for the development of SLN with desired properties. To conclude, SLNs of RLX could be an effective strategy in reducing the dose of the drug and enhancing its oral bioavailability.

\section{ACKNOWLEDGMENT}

The authors would like to thanks the Department of Pharmaceutical Sciences, Bhimtal kumaun University for providing the facility for the research. The authors would also like to thanks IIT Roorkee for their lab facility and support.

\section{FUNDING}

Nil

\section{AUTHORS CONTRIBUTIONS}

All the authors have contributed equally.

\section{CONFLICT OF INTERESTS}

Declared none

\section{REFERENCES}

1. Moen MD, Keating GM. Raloxifene: a review of its use in the prevention of invasive breast cancer. Drugs 2008;68:2059-83.
2. Jose R, Eduardo C, Maria R, Emilio C, Angel A. Raloxifene: mechanism of action, effects on bone tissue, and applicability in clinical traumatology practice. Open Orthop J 2009;3:14-21.

3. Hochner Celnikier D. Pharmacokinetics of raloxifene and its clinical application. Eur J Obstet Gynecol Reprod Biol 1999;85:23-9.

4. Jha RK, Tiwari S, Mishra B. Bioadhesive microspheres for bioavailability enhancement of raloxifene hydrochloride: formulation and pharmacokinetic evaluation. AAPS PharmSciTech 2011;12:650-7.

5. Das S, Chaudhury A. Recent advances in lipid nanoparticle formulations with solid matrix for oral drug delivery. AAPS PharmSciTech 2011;12:62-76.

6. Remya PN, Damodharan N. Formulation, development, and characterisation of cilnidipine loaded solid lipid nanoparticles. Asian J Pharm Clin Res 2018;11:120-5.

7. Sharma M, Sharma R, Jain DK. Nanotechnology-based approaches for enhancing oral bioavailability of poorly watersoluble antihypertensive drugs. Scientifica 2016;8525679. DOI:10.1155/2016/8525679

8. Ghasemiyeh P, Mohammadi Samani S. Solid lipid nanoparticles and nanostructured lipid carriers as novel drug delivery systems: applications, advantages and disadvantages. Res Pharm Sci 2018:13:288-303

9. Sharma A, Mehta V, Parashar A, Patrwal R, Malairaman U. Solid lipid nanoparticle: fabricated through nanoprecipitation and their physicochemical characterization. Int J Pharm Pharm Sci 2016;10:144-8.

10. Burra M, Jukanti R, Janga K, Sunkavalli S, Velpula A, S Ampati, et al. Enhanced intestinal absorption and bioavailability of raloxifene hydrochloride via lyophilized solid lipid nanoparticles. Adv Powder Technol 2013;24:393-402.

11. Muller R, Ruhl D, Runge S. Biodegradation of solid lipid nanoparticles as a function of lipase incubation time. Int J Pharm 1996;144:115-21.

12. Göppert T, Muller R. Adsorption kinetics of plasma proteins on solid lipid nanoparticles for drug targeting. Int J Pharm 2005;302:172-86. 
13. Castelli F, Puglia C, Sarpietro M, Rizza L, Bonina F. Characterization of indomethacin-loaded lipid nanoparticles by differential scanning calorimetry. Int J Pharm 2005;304:231-8.

14. Hao J, Fang X, Zhou Y, Wang J, Guo F. Development and optimization of solid lipid nanoparticle formulation for ophthalmic delivery of chloramphenicol using a box-behnken design. Int J Nanomed 2011;6:683-92.

15. Wang F, Chen L, Jiang S, Jin Peng, Qunwei Xu. Optimization of methazolamide-loaded solid lipid nanoparticles for ophthalmic delivery using box-behnken design.J Liposome Res 2014;24:171-81

16. Gupta B, Poudel BK, Pathak S, Tak WI, Lee HH. Effect of formulation variables on the particle size and drug encapsulation of imatinib-loaded solid-liquid nanoparticles. AAPS PharmSciTech 2016:17:652-62.

17. Prozorov T, Prozorov R, Suslick KS. High-velocity interparticle collisions driven by ultrasound.J Am Chem Soc 2004;126:13890-1.

18. Shah M, Pathak K. Development and statistical optimization of solid lipid nanoparticles of simvastatin by using 23 fullfactorial design. AAPS PharmSciTech 2010;78:1-8.

19. Yang $Y$, Chung $T$, Bai X, Chan W. Effect of preparation conditions on morphology and release profiles of biodegradable polymeric microspheres containing protein fabricated by the double-emulsion method.Chem Eng Sci 2000;55:2223-6. 